\title{
Thermophysical properties of ionic liquids and their mixtures from a new equation of state
}

\author{
F. Akbari ${ }^{1,2}$ - M. M. Alavianmehr ${ }^{1}$ - R. Behjatmanesh Ardakani ${ }^{2}$. \\ D. Mohammad-Aghaie ${ }^{1}$
}

Received: 28 May 2017 / Revised: 28 June 2017 / Accepted: 18 October 2017 / Published online: 6 November 2017

(C) The Author(s) 2017. This article is an open access publication

\begin{abstract}
In our previous work, a perturbed hard-trimersphere equation of state (PHTS EOS) was developed for modeling the phase equilibria of pure ionic liquids (ILs) (M.M. Alavianmehr et al., Ionics 22 (2016) 2447-2459). In this work, we have successfully extended the model to the mixtures of IL + IL and IL + solvent. Two temperaturedependent parameters appearing in the EOS are correlated with two microscopic scaling constants $\sigma$, the effective hardsphere diameter, and $\varepsilon$, the non-bonded interaction energy. The overall average absolute deviation (AAD) of the estimated densities from the literature data using the proposed model with and without non-additivity parameter $\left(\lambda_{\mathrm{ij}}\right)$ was found to be 0.44 and $0.79 \%$, respectively. A modified Enskog equation and rough hard-sphere (RHS) theory are combined with our proposed equation of state to calculate the viscosity coefficient of ionic liquids and their mixtures. Finally, from the results obtained, a linear relation between logarithm of surface tension and viscosity property of ionic liquid was developed.
\end{abstract}

Keywords Equation of state · Ionic liquids · Viscosity · Surface tension

\section{F. Akbari}

falamarzakbari@yahoo.com

1 Department of Chemistry, Shiraz University of Technology, Shiraz 71555-313, Iran

2 Department of Chemistry, Payame Noor University, P. O. Box, Tehran 19395-3697, Iran

\section{Introduction}

As we know, ionic liquids (ILs) are organic salts that are liquid near room temperature. They have a negligible vapor pressure under normal operating conditions and have a large liquid working temperature range. In general, ILs have many favorable properties which make them attractive for scientific community. Understanding thermophysical properties of pure ILs and their mixtures is essential to determine potential applications [1].

In recent years, some computational methods have been proposed for predicting densities of ionic liquids. Palomar et al. [2] used COSMO-RS predictive model which was based on the quantum chemistry. Group contribution (GC) and the related methods such as GC-EOS have also been widely used by numerous researchers to predict the density of ILs. Gardas and Coutinho [3] extended group contribution model to predict the density of ILs as a function of the temperature and pressure for varieties of ionic liquids. Valderama et al. [4] presented an artificial neural network and group contribution method to correlate the density of ILs. Aparicio et al. [5] reviewed the available predictive models as well as experimental data reported in literature for the thermophysical properties and especially density and PVT behavior of ionic liquids. Further, a perturbed hard-sphere equation of state has been employed to predict the pressure-volume-temperature properties of some pure and mixture of ionic liquids [6-9]. Also, they predicted the volumetric properties of ILs using Ihm-Song-Mason (ISM) [10] and Tao-Mason [11] equations of state. Hosseini et al. [12] have assessed the role of harddimers to present an alternative perturbed hard chain (PHC) 
EOS for ILs. The performance of the perturbed hard-dimer chain (PHDC) model has also been checked for density of ILs. The Tao-Mason (TM) EOS has been employed to model the pressure-volume-temperature (PVT) properties of ILs by the help of an alternative corresponding states correlation based on the measurable scaling constants, i.e., surface tension and liquid density [13]. Alavianmehr and co-workers have revised an attractive part of the preceding EOS by reevaluating the abovementioned variable parameter as well as the repulsive term. Two temperature-dependent parameters appearing in the revisited EOS have been determined from the corresponding states correlations using the interfacial properties of ILs, i.e., surface tension and liquid density, both at room temperature [14].

Recently, Alavianmehr et al. [15] developed a new version of the PHC EOS, by employing a trimer expression from the statistical associating fluid theory-trimer (SAFT-T) as the reference physical model. The perturbation part of the proposed EOS was taken from the van der Waals dispersion forces. The proposed model was employed to predict the volumetric properties of polymers. In our previous work [16], we examined the predictive power of this EOS for the prediction of the liquid density, isothermal compressibilities, and thermal expansion coefficients as well as the heat capacities and vapor pressure of pure ILs. For this purpose, 39 ILs with various anions were chosen. Our calculation results were summarized as average absolute deviation percent (AAD\%) from the literature data. From 6331 data points examined for the studied pure ILs over a broad pressures ranging from 0.1 to $200 \mathrm{MPa}$ and temperatures ranging from 273 to $472.6 \mathrm{~K}$, the AAD was found to be $0.18 \%$.

In the present study, this model is successfully applied to predict thermophysical properties of mixtures containing ionic liquids. Thermophysical properties consist of densities, surface tensions, and viscosity coefficients.

Liquid viscosity is an important transport property in chemical process design. Therefore, prediction of the viscosity coefficient of ionic liquids is a very useful task. Starting from the pioneering work of Hsu and Eyring [17] on the application of the significant structure theory to the calculation of the viscosity of liquid alkali metals, other theoretical prediction methods have been developed so far for this purpose. The temperature dependence of transport properties of fluids is also essential for the most industrial applications, and intensive research has been done for their measurement [18-20]. Fluid viscosity, among other transport properties, can be measured basically with high accuracy. Experimental viscosity of dilute gases and its temperature dependence are used as essential properties for accurate determination of molecular information such as pair interaction potential function. These properties accurately furnish the basic information of fluids so that their correlations provide a good insight into different fluid classes. In this work, we propose a correlation for the temperature and density dependence of the viscosity coefficient of ionic liquids. The correlation has a similar mathematical expression as the modified Enskog theory expression [21] for the viscosity of dense fluids. It is shown that the present approach can well predict the viscosity coefficient of ionic liquids within a few percent of experimental measurements. In the present work, the liquid viscosity for ILs is predicted using the PHTS model in conjunction with the modified Enskog method and rough hardsphere (RHS) theory [22].

Liquid surface tension as an equilibrium thermodynamic property is also as important as viscosity in the design of industrial applications. Surface tension of a liquid is related to the intermolecular interaction potential energy and the liquid interfacial microstructure; it decreases linearly with temperature in the range of freezing to the boiling temperature and vanishes non-linearly close to the critical point [23-26]. Like viscosity, surface tension can be measured with high accuracy. The temperature dependence of surface tension, the negative surface entropy, can be used to characterize fluids in terms of the molecular energetics and surface microstructure. Contrary to viscosity, the surface tension decreases very smoothly with temperature; its dependence on temperature is constant and close to one another for most normal liquids [27, 28].

In the present study, we have evaluated PHTS EOS by predicting the surface tension for ionic liquids. The thermophysical properties of mixtures are important because they depend on the composition and/or temperature and are of great importance in understanding the nature of molecular aggregation that exists in the binary mixtures.

\section{Theory}

\section{Equation of state for single substance}

The general form of PHTS EOS is as follows:

$Z=Z^{H 3}+Z^{\text {pert }}$

In Eq. (1), the reference equation takes the form proposed by Srivastava and Khanna [29]. The formalism mentioned here is based on the assumption that the chain formed by the pairs of trimers:

$Z^{H 3}=3 Z_{M V}^{H S}-2\left\{1+y \frac{\partial \ln \left(\left(Z_{M V}^{H S}-1\right) / 4 y\right)}{\partial y}\right\}$

The reference system is the new version of the hard-sphere (HS) model expressed by Malijevsky and Veverka (MV) equation [30]:

$Z_{M V}^{H S}=\frac{1+1.056 y+1.6539 y^{2}+0.3262 y^{3}}{\left(1+0.056 y+0.5979 y^{2}+0.3076 y^{3}\right)(1-y)^{3}}$ 
where $y$ is the packing fraction of hard-core which is defined as:

$y=\frac{b(T) \rho}{4}$

The perturbed term used in this work is van der Waals attractive form:

$Z^{\text {pert }}=-\frac{a(T) \rho}{k_{B} T}$

The two temperature-dependent parameters $a(T)$ and $b(T)$ can be expressed in terms of the universal function of reduced temperature according to the law of corresponding states principle. They are correlated with the hardsphere diameter, $\sigma$, and the non-bonded interaction energy between two trimer spheres, $\varepsilon$, according to the following equations:

$a(T)=\frac{2 \pi}{3} \sigma^{3} \varepsilon F_{a}\left(\frac{k_{B} T}{\varepsilon}\right)$

$b(T)=\frac{2 \pi}{3} \sigma^{3} F_{b}\left(\frac{k_{B} T}{\varepsilon}\right)$

where $F_{a}$ and $F_{b}$ are the universal functions of the reduced temperature $\left(k_{B} T / \varepsilon\right)$, which can be written as the following formula:

$$
\begin{aligned}
F_{a}\left(k_{B} T / \varepsilon\right)= & 1.058393 \exp \left[-0.879380\left(k_{B} T / \varepsilon\right)\right] \\
& +0.609445 \exp \left[-0.314038\left(k_{B} T / \varepsilon\right)^{3 / 2}\right] \\
F_{b}\left(k_{B} T / \varepsilon\right)= & 0.798019 \exp \left[0.908086\left(k_{B} T / \varepsilon\right)\right] \\
& +0.521304 \exp \left[-0.407266\left(k_{B} T / \varepsilon\right)^{3 / 2}\right]
\end{aligned}
$$

\section{Mixture version of the model}

The mixture version of the proposed equation of state can be written as below:

$$
\begin{aligned}
Z_{m} & =3 Z_{M V, m}^{H S}-2\left\{1+y_{m} \frac{\partial \ln \left(\left(Z_{M V, m}^{H S}-1\right) / 4 y_{m}\right)}{\partial y_{m}}\right\} \\
& -\frac{\rho}{R T} \sum_{i j}^{m} x_{i} x_{j} a(T)_{i j}
\end{aligned}
$$

where $x_{i}$ and $x_{j}$ are the mole fractions of $i$ th and $j$ th components, respectively. $\eta_{m}$ is the packing fraction of the mixtures of hard-spheres [31]. This parameter is defined by the following expression:

$y_{m}=\frac{\rho}{4} \sum_{i} x_{i} b(T)_{i}$

The compressibility factor of hard-sphere mixtures takes the form:

$Z_{M V, m}^{H S}=\frac{1+1.056 y_{m}+1.6539 y_{m}^{2}+0.3262 y_{m}^{3}}{\left(1+0.056 y_{m}+0.5979 y_{m}^{2}+0.3076 y_{m}^{3}\right)\left(1-y_{m}\right)^{3}}$

The attractive forces between two hard-sphere species of a mixture including $i$ and $j$ components can be written as follows:

$a(T)_{i j}=\frac{2 \pi}{3} \sigma_{i j}^{3} \varepsilon_{i j} F_{a}\left(\frac{k_{B} T}{\varepsilon}\right)_{i j}$

The present method for calculating two temperaturedependent parameters can be extended to mixtures by using simple geometric and arithmetic mean for the adjustable scaling constants, i.e.,

$\varepsilon_{i j}=\left(1-\lambda_{i j}\right) \sqrt{\left(\varepsilon_{i} \varepsilon_{j}\right)}$

and

$\sigma_{i j}=\frac{1}{2}\left(\sigma_{i}+\sigma_{j}\right)$

where $\lambda_{i j}$ is a binary interaction parameter which shows non-additivity of the mixture. Further, the universal function $F$ for $i j$ mixture is proposed to be obtained by the following geometric mean from its corresponding ones for pure substances, i.e., Eqs. (8) and (9):

$F_{a}\left(\frac{k_{B} T}{\varepsilon}\right)_{i j}=\sqrt{F_{a}\left(k_{B} T / \varepsilon\right)_{i} F_{a}\left(k_{B} T / \varepsilon\right)_{j}}$

\section{Results and discussion}

In previous section, a new equation of state was developed to model thermophysical properties of ILs and their binary mixtures. To utilize the proposed PHTS EOS, the values of two pure component parameters, $\sigma$ and $\varepsilon$, of studied ILs and solvents must be characterized. Physically, theses parameters reflect the hard-core 
diameter and non-bonded interaction energy between pair-wise trimers, respectively. In this work, these parameters were optimized for each ILs and solvents by fitting the proposed model with experimental densities data. To achieve this goal, the following objective function was minimized using the least-squares method:

$O F=\min \frac{1}{n} \sum_{i=1}^{n} \frac{\left|M_{\mathrm{i}}^{\text {Lit. }}-M_{\mathrm{i}}^{\text {Cal. }}\right|}{M_{\mathrm{i}}^{\text {Lit. }}}$

where $M$ and $n$ represent thermodynamic properties and the number of relevant density data points for a given ILs and/or solvents, respectively. The numerical values of optimized pure component parameters of studied fluids are reported in Table 1. Generally, from 1927 literature data points applied to nine studied ILs (cited in Table 1), the minimum value of $\mathrm{OF}$ was found to be 0.080 .

In the present study, the proposed model is extended to various binary mixtures formed by ILs and molecular solvents including water, ethanol, methanol, 1-propanol, THF, and acetonitrile using some mixing and combining rules. Table 2 tabulates the AAD of the predicted densities of several binary of IL + IL and IL + solvent mixtures at various temperatures and mole fractions from literature values [32-39]. Besides, the maximum deviations (MD) of the calculations have also been included in Table 2. It should be mentioned that the maximum deviation of the calculated volumetric properties of all binary mixtures studied in this work was found to be $3.46 \%$. It is noticeable that the uncertainty of the calculated density for all binary mixtures studied in this work was of the order of \pm 1.55 in temperature range $278-358 \mathrm{~K}$ and atmospheric pressure.

In the current work, the non-additivity effect on our proposed model has also been investigated. Table 2 lists the results with and without the non-additivity effect. The AADs of the predicted mixture densities for 1927 data points using the present model were found to be 0.44 and $0.79 \%$ with and without adjustable parameter $\left(\lambda_{i j}\right)$, respectively. As Table 2 shows, the numerical values of
Table 1 The scaling pure component parameters to be used in Eqs. (4), (5) and (18)

\begin{tabular}{|c|c|c|c|c|c|c|}
\hline & $M_{\mathrm{W}}\left(\mathrm{g} \mathrm{mol}^{-1}\right)$ & $\varepsilon / k_{\mathrm{B}}(\mathrm{K})$ & $\sigma(\mathrm{nm})$ & $\alpha$ (mPas) & $\beta$ & $\omega$ \\
\hline \multicolumn{7}{|l|}{ Ionic liquid } \\
\hline$\left[\mathrm{C}_{2} \mathrm{mim}\right][$ Triflat $]$ & 260.23 & 4833.7 & 0.6382 & 1271.530 & -7.907 & 247.511 \\
\hline$\left[\mathrm{C}_{4} \operatorname{mim}\right][$ Triflat $]$ & 288.29 & 7106.3 & 0.6917 & 1544.080 & -11.832 & 554.110 \\
\hline$\left[\mathrm{C}_{2} \mathrm{mim}\right]\left[\mathrm{EtSO}_{4}\right]$ & 236.29 & 7465.0 & 0.6595 & 5162.113 & -13.470 & 720.392 \\
\hline$\left[\mathrm{C}_{4} \operatorname{mim}\right]\left[\mathrm{C}(\mathrm{CN})_{3}\right]$ & 229.28 & 5010.5 & 0.6716 & 883.100 & -8.080 & 259.506 \\
\hline$\left[\mathrm{C}_{4} \operatorname{mim}\right]\left[\mathrm{N}(\mathrm{CN})_{2}\right]$ & 205.20 & 7950.0 & 0.6652 & 940.500 & -13.609 & 735.645 \\
\hline$\left[\mathrm{C}_{4} \mathrm{mim}\right]\left[\mathrm{MeSO}_{4}\right]$ & 250.32 & 6355.0 & 0.6703 & $18,526.398$ & -11.450 & 522.002 \\
\hline$\left[\mathrm{C}_{2} \operatorname{mim}\right]\left[\mathrm{NTf}_{2}\right]$ & 391.31 & 5539.7 & 0.7144 & 656.641 & -9.014 & 320.230 \\
\hline$\left[\mathrm{C}_{4} \operatorname{mim}\right]\left[\mathrm{NTf}_{2}\right]$ & 419.36 & 5919.0 & 0.7486 & 1313.646 & -9.817 & 381.816 \\
\hline$\left[\mathrm{C}_{6} \operatorname{mim}\right]\left[\mathrm{NTf}_{2}\right]$ & 447.42 & 5735.8 & 0.7747 & 1991.562 & -9.498 & 358.139 \\
\hline$\left[\mathrm{C}_{8} \operatorname{mim}\right]\left[\mathrm{NTf}_{2}\right]$ & 475.47 & 5335.2 & 0.7975 & 2882.353 & -8.766 & 305.382 \\
\hline$\left[\mathrm{C}_{10} \mathrm{mim}\right]\left[\mathrm{NTf}_{2}\right]$ & 503.52 & 5627.2 & 0.8249 & 2491.448 & -9.096 & 328.596 \\
\hline$\left[\mathrm{C}_{2} \mathrm{mim}\right]\left[\mathrm{BF}_{4}\right]$ & 197.97 & 2938.8 & 0.6052 & 1147.998 & -9.091 & 327.337 \\
\hline$\left[\mathrm{C}_{3} \mathrm{mim}\right]\left[\mathrm{BF}_{4}\right]$ & 211.97 & 3940.0 & 0.6069 & 2047.131 & -6.167 & 150.845 \\
\hline$\left[\mathrm{C}_{4} \mathrm{mim}\right]\left[\mathrm{BF}_{4}\right]$ & 226.02 & 5391.4 & 0.6410 & 2000.958 & -8.659 & 297.645 \\
\hline$\left[\mathrm{C}_{6} \mathrm{mim}\right]\left[\mathrm{BF}_{4}\right]$ & 254.08 & 5220.0 & 0.6766 & 7796.281 & -8.640 & 297.215 \\
\hline$\left[\mathrm{C}_{8} \mathrm{mim}\right]\left[\mathrm{BF}_{4}\right]$ & 282.13 & 5598.0 & 0.7132 & 5275.015 & -8.785 & 307.319 \\
\hline$\left[\mathrm{C}_{4} \mathrm{mim}\right]\left[\mathrm{PF}_{6}\right]$ & 284.18 & 5005.0 & 0.6605 & 3922.981 & -7.657 & 233.157 \\
\hline$\left[\mathrm{C}_{6} \mathrm{mim}\right]\left[\mathrm{PF}_{6}\right]$ & 312.24 & 5406.0 & 0.6973 & 8438.436 & -8.466 & 285.631 \\
\hline$\left[\mathrm{C}_{8} \operatorname{mim}\right]\left[\mathrm{PF}_{6}\right]$ & 340.29 & 7292.0 & 0.7731 & $61,055.050$ & -13.210 & 696.093 \\
\hline \multicolumn{7}{|l|}{ Solvent } \\
\hline Ethanol & 46 & 1690.0 & 0.38605 & 4.123 & -1.966 & 14.002 \\
\hline Water & 18 & 6720.0 & 0.30055 & 4.971 & -10.308 & 400.453 \\
\hline Methanol & 32 & 1650.0 & 0.33955 & - & - & - \\
\hline 1-Propanol & 60 & 2680.0 & 0.44405 & - & - & - \\
\hline Acetonitrile & 41.05 & 1765.0 & 0.37555 & - & - & - \\
\hline THF & 72.11 & 1550.0 & 0.42550 & - & - & - \\
\hline
\end{tabular}

$M_{\mathrm{W}}$ molar weight 
Table 2 AAD of the predicted densities of binary mixtures including IL + solvent and IL + IL using the proposed model from the literature ones

\begin{tabular}{|c|c|c|c|c|c|c|c|}
\hline Mixture & $T_{\min }-T_{\max }(\mathrm{K})$ & NP & $\lambda_{i j}$ & $\mathrm{AAD}^{\mathrm{a}} \%$ & $\mathrm{AAD}^{\mathrm{b}} \%$ & MD \% & Ref. \\
\hline \multirow[t]{2}{*}[\mathrm{C}_{2}\mathrm{mim}]{$\left[\mathrm{BF}_{4}\right]+\left[\mathrm{C}_{6} \mathrm{mim}\right]\left[\mathrm{BF}_{4}\right]$} & $298-308$ & 135 & \multirow[t]{2}{*}{0.06} & 0.33 & 0.77 & 0.96 & {$[32]$} \\
\hline & $293-343$ & 121 & & 0.39 & 0.60 & 1.19 & [39] \\
\hline$\left[\mathrm{C}_{2} \mathrm{mim}\right]\left[\mathrm{BF}_{4}\right]+\left[\mathrm{C}_{3} \mathrm{mim}\right]\left[\mathrm{BF}_{4}\right]$ & $293-343$ & 121 & 0.04 & 0.35 & 0.45 & 0.98 & [39] \\
\hline$\left[\mathrm{C}_{3} \mathrm{mim}\right]\left[\mathrm{BF}_{4}\right]+\left[\mathrm{C}_{6} \mathrm{mim}\right]\left[\mathrm{BF}_{4}\right]$ & $293-343$ & 121 & 0.04 & 0.41 & 0.56 & 1.19 & [39] \\
\hline$\left[\mathrm{C}_{4} \mathrm{mim}\right]\left[\mathrm{BF}_{4}\right]+\left[\mathrm{C}_{4} \mathrm{mim}\right]\left[\mathrm{MeSO}_{4}\right]$ & $298-308$ & 144 & 0.04 & 0.18 & 0.46 & 0.62 & [32] \\
\hline$\left[\mathrm{C}_{4} \mathrm{mim}\right]\left[\mathrm{PF}_{6}\right]+\left[\mathrm{C}_{4} \mathrm{mim}\right]\left[\mathrm{BF}_{4}\right]$ & $298-308$ & 126 & 0.011 & 0.23 & 0.27 & 0.62 & [32] \\
\hline$\left[\mathrm{C}_{4} \mathrm{mim}\right]\left[\mathrm{N}(\mathrm{CN})_{2}\right]+$ water & $278-358$ & 63 & -0.05 & 0.084 & 0.29 & 0.37 & [37] \\
\hline$\left[\mathrm{C}_{4} \mathrm{mim}\right]\left[\mathrm{BF}_{4}\right]+$ water & $308-353$ & 88 & 0.11 & 0.42 & 1.35 & -2.81 & [33] \\
\hline$\left[\mathrm{C}_{4} \mathrm{mim}\right][$ Triflate $]+$ water & $303-343$ & 77 & -0.05 & 0.36 & 0.71 & -2.81 & [34] \\
\hline$\left[\mathrm{C}_{2} \mathrm{mim}\right]\left[\mathrm{EtSO}_{4}\right]+$ water & $278-308$ & 112 & -0.09 & 0.83 & 1.01 & -2.26 & {$[35]$} \\
\hline$\left[\mathrm{C}_{2} \mathrm{mim}\right][$ Triflate $]+$ water & $278-338$ & 133 & 0.08 & 0.38 & 1.03 & -1.91 & [36] \\
\hline$\left[\mathrm{C}_{4} \mathrm{mim}\right]\left[\mathrm{C}(\mathrm{CN})_{3}\right]+$ water & $278-358$ & 81 & 0.12 & 0.20 & 1.08 & -0.51 & [37] \\
\hline$\left[\mathrm{C}_{2} \mathrm{mim}\right]\left[\mathrm{EtSO}_{4}\right]+$ methanol & $278-308$ & 147 & 0.01 & 0.95 & 0.95 & 3.46 & [35] \\
\hline$\left[\mathrm{C}_{2} \mathrm{mim}\right]\left[\mathrm{EtSO}_{4}\right]+$ ethanol & $278-308$ & 126 & 0.13 & 0.47 & 0.99 & 1.43 & [35] \\
\hline$\left[\mathrm{C}_{2} \mathrm{mim}\right][$ Triflate $]+$ ethanol & $278-328$ & 60 & 0.16 & 0.73 & 2.00 & 3.12 & [36] \\
\hline$\left[\mathrm{C}_{2} \mathrm{mim}\right][$ Triflate $]+1$-propanol & $278-338$ & 91 & 0.01 & 0.29 & 0.29 & 0.59 & [36] \\
\hline$\left[\mathrm{C}_{2} \mathrm{mim}\right]\left[\mathrm{EtSO}_{4}\right]+$ acetonitrile & $278-308$ & 168 & 0.05 & 0.67 & 0.74 & -1.89 & [35] \\
\hline$\left[\mathrm{C}_{4} \mathrm{mim}\right]\left[\mathrm{PF}_{6}\right]+\mathrm{THF}$ & 298 & 13 & 0.01 & 0.62 & 0.62 & 1.10 & [38] \\
\hline Overall & & 1927 & & 0.44 & 0.79 & & \\
\hline
\end{tabular}

$\mathrm{AAD}=100 / \mathrm{NP} \sum_{\mathrm{i}=1}^{\mathrm{NP}}\left|\rho_{\mathrm{i}}^{\text {Calc. }}-\rho_{\mathrm{i}}^{\text {Lit. }}\right| / \rho_{\mathrm{i}}^{\text {Lit. }}$

$N P$ number of literature data points examined, $M D$ maximum deviation of the calculated $\rho_{\text {mix }}$

${ }^{\mathrm{a}}$ The present model with adjustable parameter

${ }^{\mathrm{b}}$ The present model without adjustable parameter

the non-additivity parameter $\left(\lambda_{i j}\right)$ for the studied mixtures are nearly low. A nonzero non-additivity parameter indicates some interaction between the unlike components. The low values of this parameter are in favor of this fact that the volumes of ionic liquids are nearly additive. However, considering non-additivity effect improves slightly the results of the calculated densities of IL mixtures.

In general, a wide space of experimental data points regarding the binary mixtures has been taken to check the predictive power of the proposed EOS. To show how the mixture version of our model passes through the experimental points, Fig. 1 has been provided. Figure 1 shows the calculated and literature values of densities of binary mixtures $\left[\mathrm{C}_{2} \mathrm{mim}\right][$ Triflate $]+$ 1-Propanol at different temperatures.

To ascertain the degree of reliability of the present model, we compare the correlated and predicted results from the proposed model with other models given in literature. In this respect, we compare the outcomes of our calculations with those obtained from Fadaei-Nobandegani et al [40]. Table 3 contains the AAD of the calculated densities of binary mixtures using the present model and other equations of state [40]

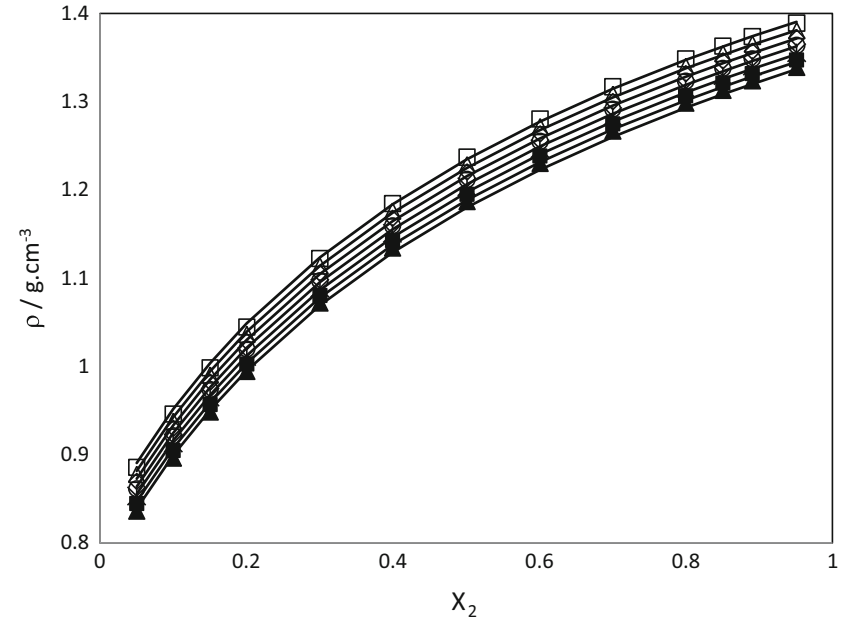

Fig. 1 Density of $\left[\mathrm{C}_{2}\right.$ mim $][$ Triflate $]\left(\mathrm{x}_{1}\right)+1$-Propanol $\left(x_{2}\right)$ as a function of mole fraction $(x)$ of 1-Propanol at different temperatures and pressures (with adjustable parameter). The markers represent the literature data [36], $278.15 \mathrm{~K}$ (white square); $288.15 \mathrm{~K}$ (white up-pointing triangle), 298.15 K (white diamond), $308.15 \mathrm{~K}$ (white circle), $318.15 \mathrm{~K}$ (asterisk), $328.15 \mathrm{~K}$ (black square), and $338.15 \mathrm{~K}$ (black up-pointing triangle), while the solid curves represent the results calculated with the proposed model 
Table 3 Comparison of the AAD of calculated density of binary mixtures of ILs (IL + IL and IL + solvent) in this work and those obtained by PHS EOS [40] compared with the literature data [32-36]

\begin{tabular}{|c|c|c|c|c|c|}
\hline & $T_{\min }-T_{\max }(\mathrm{K})$ & NP & This work & PHS & Ref. \\
\hline$\left[\mathrm{C}_{2} \mathrm{mim}\right]\left[\mathrm{BF}_{4}\right]+\left[\mathrm{C}_{6} \mathrm{mim}\right]\left[\mathrm{BF}_{4}\right]$ & $298-308$ & 135 & 0.33 & 1.20 & [32] \\
\hline$\left[\mathrm{C}_{4} \mathrm{mim}\right]\left[\mathrm{PF}_{6}\right]+\left[\mathrm{C}_{4} \mathrm{mim}\right]\left[\mathrm{BF}_{4}\right]$ & $298-308$ & 126 & 0.23 & 1.14 & [32] \\
\hline$\left[\mathrm{C}_{4} \operatorname{mim}\right]\left[\mathrm{BF}_{4}\right]+$ water & $308-353$ & 88 & 0.42 & 0.89 & [33] \\
\hline$\left[\mathrm{C}_{4} \mathrm{mim}\right][$ Triflate $]+$ water & $303-343$ & 77 & 0.36 & 0.68 & [34] \\
\hline$\left[\mathrm{C}_{2} \mathrm{mim}\right]\left[\mathrm{EtSO}_{4}\right]+$ water & $278-308$ & 112 & 0.83 & 0.65 & [35] \\
\hline$\left[\mathrm{C}_{2} \mathrm{mim}\right][$ Triflate $]+$ water & $278-338$ & 133 & 0.38 & 0.48 & [36] \\
\hline$\left[\mathrm{C}_{2} \mathrm{mim}\right]\left[\mathrm{EtSO}_{4}\right]+$ methanol & $278-308$ & 147 & 0.95 & 0.39 & {$[35]$} \\
\hline Overall & & 818 & 0.50 & 0.78 & \\
\hline
\end{tabular}

$\mathrm{AAD}=100 / \mathrm{NP} \sum_{i=1}^{\mathrm{NP}}\left|\rho_{i}^{\text {Calc. }}-\rho_{i}^{\mathrm{Lit}}\right| / \rho_{i}^{\mathrm{Lit}}$

$N P$ number of literature data points examined

from the literature data [32-36]. Close inspection of the results shows the ability of the present model to describe liquid density data for IL mixtures. Interestingly, the results are in favor of superiority of the present model over the one proposed in literature [40].

The proposed model was further evaluated by prediction of other thermophysical properties including viscosity and surface tension.

\section{Viscosity}

\section{Modified Enskog theory}

According to the modified Enskog theory (MET) [21], the viscosity coefficient of a dense gas of rigid spherical molecules is related to pair distribution function, density, and dilute gas viscosity. As we know, MET is applicable to dense gases and
Table 4 The coefficients $c_{1}-c_{3}$ for each IL and solvent to be used in Eq. (22) obtained from the viscosity data at pressure $0.1 \mathrm{MPa}$

\begin{tabular}{llll}
\hline & $c_{1}$ & $c_{2}$ & $c_{3}$ \\
\hline Ionic liquid & & & \\
{$\left[\mathrm{C}_{2} \mathrm{mim}\right][$ Triflat $]$} & $3.4167 \times 10^{-05}$ & $-9.9008 \times 10^{-04}$ & $7.2170 \times 10^{-03}$ \\
{$\left[\mathrm{C}_{4} \mathrm{mim}\right][$ Triflat $]$} & $2.4070 \times 10^{-05}$ & $-9.8118 \times 10^{-04}$ & $1.0069 \times 10^{-02}$ \\
{$\left[\mathrm{C}_{2} \mathrm{mim}\right]\left[\mathrm{EtSO}_{4}\right]$} & $6.8953 \times 10^{-05}$ & $-3.1636 \times 10^{-03}$ & $3.6476 \times 10^{-02}$ \\
{$\left[\mathrm{C}_{4} \mathrm{mim}\right]\left[\mathrm{C}(\mathrm{CN})_{3}\right]$} & $1.8434 \times 10^{-05}$ & $-5.4093 \times 10^{-04}$ & $3.9811 \times 10^{-03}$ \\
{$\left[\mathrm{C}_{4} \mathrm{mim}\right]\left[\mathrm{N}(\mathrm{CN})_{2}\right]$} & $1.2583 \times 10^{-05}$ & $-5.8308 \times 10^{-04}$ & $6.7837 \times 10^{-03}$ \\
{$\left[\mathrm{C}_{4} \mathrm{mim}\right]\left[\mathrm{MeSO}_{4}\right]$} & $2.6713 \times 10^{-04}$ & $-1.0623 \times 10^{-02}$ & $1.0596 \times 10^{-01}$ \\
{$\left[\mathrm{C}_{2} \mathrm{mim}\right]\left[\mathrm{NTf}_{2}\right]$} & $1.3882 \times 10^{-05}$ & $-4.4431 \times 10^{-04}$ & $3.5898 \times 10^{-03}$ \\
{$\left[\mathrm{C}_{4} \mathrm{mim}\right]\left[\mathrm{NTf}_{2}\right]$} & $2.3726 \times 10^{-05}$ & $-8.1998 \times 10^{-04}$ & $7.1295 \times 10^{-03}$ \\
{$\left[\mathrm{C}_{6} \mathrm{mim}\right]\left[\mathrm{NTf}_{2}\right]$} & $3.5197 \times 10^{-05}$ & $-1.1835 \times 10^{-03}$ & $9.9993 \times 10^{-03}$ \\
{$\left[\mathrm{C}_{8} \mathrm{mim}\right]\left[\mathrm{NTf}_{2}\right]$} & $5.2316 \times 10^{-05}$ & $-1.6415 \times 10^{-03}$ & $1.2931 \times 10^{-02}$ \\
{$\left[\mathrm{C}_{10} \mathrm{mim}\right]\left[\mathrm{NTf}_{2}\right]$} & $4.4995 \times 10^{-05}$ & $-1.4625 \times 10^{-03}$ & $1.1938 \times 10^{-02}$ \\
{$\left[\mathrm{C}_{2} \mathrm{mim}\right]\left[\mathrm{BF}_{4}\right]$} & $1.5683 \times 10^{-05}$ & $-4.3050 \times 10^{-04}$ & $2.9816 \times 10^{-03}$ \\
{$\left[\mathrm{C}_{3} \mathrm{mim}\right]\left[\mathrm{BF}_{4}\right]$} & $5.8411 \times 10^{-05}$ & $-1.3501 \times 10^{-03}$ & $7.8424 \times 10^{-03}$ \\
{$\left[\mathrm{C}_{4} \mathrm{mim}\right]\left[\mathrm{BF}_{4}\right]$} & $4.2444 \times 10^{-05}$ & $-1.3238 \times 10^{-03}$ & $1.0374 \times 10^{-02}$ \\
{$\left[\mathrm{C}_{6} \mathrm{mim}\right]\left[\mathrm{BF}_{4}\right]$} & $1.4511 \times 10^{-04}$ & $-4.6621 \times 10^{-03}$ & $3.7552 \times 10^{-02}$ \\
{$\left[\mathrm{C}_{4} \mathrm{mim}\right]\left[\mathrm{PF}_{6}\right]$} & $1.1141 \times 10^{-04}$ & $-3.1130 \times 10^{-03}$ & $2.1824 \times 10^{-02}$ \\
{$\left[\mathrm{C}_{6} \mathrm{mim}\right]\left[\mathrm{PF}_{6}\right]$} & $1.6786 \times 10^{-04}$ & $-5.1185 \times 10^{-03}$ & $3.9110 \times 10^{-02}$ \\
{$\left[\mathrm{C}_{8} \mathrm{mim}\right]\left[\mathrm{PF}_{6}\right]$} & $6.7529 \times 10^{-04}$ & $-3.0489 \times 10^{-02}$ & $3.4482 \times 10^{-01}$ \\
Solvent $^{\mathrm{Ethanol}}$ & & & \\
Water & $5.7766 \times 10^{-07}$ & $-5.1388 \times 10^{-06}$ & $1.1890 \times 10^{-05}$ \\
\hline & $1.4248 \times 10^{-07}$ & $-4.9829 \times 10^{-06}$ & $4.5083 \times 10^{-05}$ \\
\hline & & &
\end{tabular}


therefore cannot be applied to liquids. Therefore, we take the simple form of MET and propose the following equation for the viscosity of liquids:

$\eta=\alpha\left\{1+\beta\left(b(T) \rho\left(Z_{M V}^{H S}-1\right) / 4 y\right)+\omega\left(b(T) \rho\left(Z_{M V}^{H S}-1\right) / 4 y\right)^{2}\right\}$

where $\alpha, \beta$, and $\omega$ are adjustable parameters. The experimental viscosities over wide range of temperatures and pressures were used to fix aforementioned adjustable parameters. The values of $\alpha, \beta$, and $\omega$ are reported in Table 1.

\section{Rough hard-sphere-based model}

The proposed RHS model for transport properties of ILs can be expressed as [22]:

$\eta_{R H S}^{*}=C_{\eta} \eta_{S H S-M E T}^{*}=C_{\eta} \frac{\left(M_{w} \varepsilon / N_{a}\right)^{1 / 2}}{\sigma^{2} F_{\eta}} \eta_{S H S-M E T}$

Table 5 AAD $(\%)$ of the calculated viscosity of ionic liquids using the MET [21] and RHS [22] models from the experimental data [33, 34, 37, $39,41-48]$

$$
T_{\min }-T_{\max }(\mathrm{K}) \quad \mathrm{NP} \quad \mathrm{MET} \quad \text { RHS } \quad \text { Ref }
$$

Pure

\begin{tabular}{llllll}
{$\left[\mathrm{C}_{2}\right.$ mim $]\left[\mathrm{BF}_{4}\right]$} & $293-343$ & 11 & 2.14 & 2.48 & {$[39]$} \\
{$\left[\mathrm{C}_{3}\right.$ mim $]\left[\mathrm{BF}_{4}\right]$} & $293-343$ & 11 & 4.56 & 4.90 & {$[39]$} \\
{$\left[\mathrm{C}_{6}\right.$ mim $]\left[\mathrm{BF}_{4}\right]$} & $293-343$ & 11 & 8.20 & 9.06 & {$[39]$} \\
{$\left[\mathrm{C}_{4}\right.$ mim $]\left[\mathrm{BF}_{4}\right]$} & $303-343$ & 07 & 2.13 & 2.71 & {$[33]$} \\
{$\left[\mathrm{C}_{4}\right.$ mim $]\left[\mathrm{C}(\mathrm{CN})_{3}\right]$} & $278-358$ & 09 & 17.18 & 22.20 & {$[37]$} \\
{$\left[\mathrm{C}_{4}\right.$ mim $]\left[\mathrm{N}(\mathrm{CN})_{2}\right]$} & $278-358$ & 09 & 15.88 & 17.91 & {$[37]$} \\
{$\left[\mathrm{C}_{4}\right.$ mim $]\left[\mathrm{MeSO}_{4}\right]$} & $293-313$ & 05 & 0.87 & 0.88 & {$[42]$} \\
{$\left[\mathrm{C}_{10}\right.$ mim $]\left[\mathrm{NTf}_{2}\right]$} & $298-343$ & 03 & 0.026 & 0.83 & {$[43]$} \\
{$\left[\mathrm{C}_{8}\right.$ mim $]\left[\mathrm{NTf}_{2}\right]$} & $293-343$ & 06 & 6.68 & 7.13 & {$[44]$} \\
{$\left[\mathrm{C}_{6}\right.$ mim $]\left[\mathrm{NTf}_{2}\right]$} & $293-343$ & 06 & 5.56 & 6.08 & {$[44]$} \\
{$\left[\mathrm{C}_{4}\right.$ mim $]\left[\mathrm{NTf}_{2}\right]$} & $293-343$ & 06 & 4.43 & 4.89 & {$[44]$} \\
{$\left[\mathrm{C}_{2}\right.$ mim $]\left[\mathrm{NTf}_{2}\right]$} & $293-343$ & 06 & 2.66 & 3.10 & {$[44]$} \\
{$\left[\mathrm{C}_{2}\right.$ mim $][\mathrm{Triflat}]$} & $278-338$ & 07 & 4.55 & 6.85 & {$[48]$} \\
{$\left[\mathrm{C}_{4}\right.$ mim $][\mathrm{Triflat}]$} & $303-343$ & 07 & 1.38 & 1.57 & {$[34]$} \\
{$\left[\mathrm{C}_{4}\right.$ mim $]\left[\mathrm{PF}_{6}\right]$} & $303-363$ & 07 & 8.15 & 8.86 & {$[41]$} \\
{$\left[\mathrm{C}_{6}\right.$ mim $]\left[\mathrm{PF}_{6}\right]$} & $303-363$ & 07 & 14.71 & 15.42 & {$[41]$} \\
{$\left[\mathrm{C}_{8}\right.$ mim $]\left[\mathrm{PF}_{6}\right]$} & $293-323$ & 07 & 4.66 & 4.80 & {$[45]$} \\
{$\left[\mathrm{C}_{2}\right.$ mim $]\left[\mathrm{EtSO}_{4}\right]$} & $298-313$ & 04 & 0.29 & 0.35 & {$[46]$} \\
Ethanol & $283-343$ & 13 & 0.41 & 0.49 & {$[46]$} \\
Water & $303-353$ & 08 & 0.54 & 0.61 & {$[33]$} \\
Overall & & 150 & 5.25 & 6.06 & \\
\hline & & & & &
\end{tabular}

$\mathrm{AAD}=100 / \mathrm{NP} \sum_{i=1}^{\mathrm{NP}}\left|\eta_{i}^{\text {Calc. }}-\eta_{i}^{\text {Lit. }}\right| / \eta_{i}^{\text {Lit. }}$

$N P$ number of literature data points examined, MET modified Enskog theory [21], RHS rough hard-sphere [22]

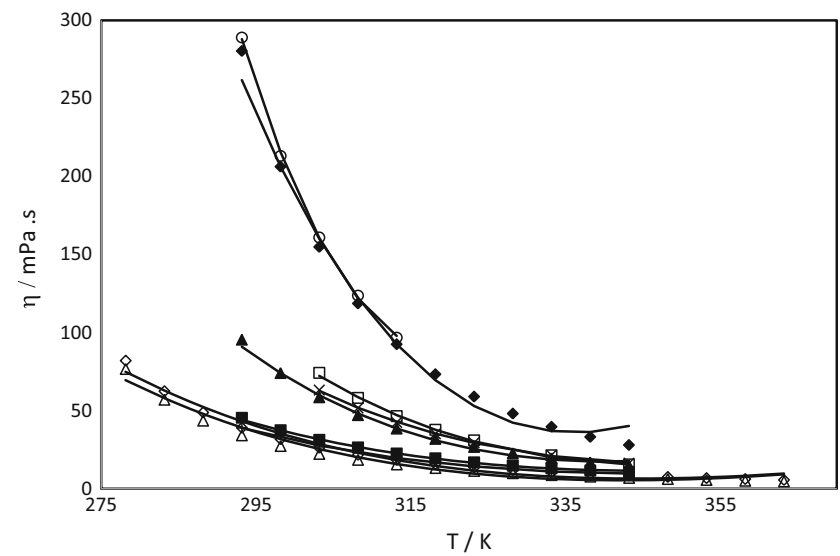

Fig. 2 Linear dependency viscosity of some selected ionic liquids on the temperature curve. The markers represent the literature data $[33,34,37$, $39,42,44],\left[\mathrm{C}_{4} \mathrm{mim}\right]\left[\mathrm{BF}_{4}\right]$ (white square), $\left[\mathrm{C}_{4} \mathrm{mim}\right]\left[\mathrm{C}(\mathrm{CN})_{3}\right]$ (white uppointing triangle), $\left[\mathrm{C}_{4} \mathrm{mim}\right]\left[\mathrm{N}(\mathrm{CN})_{2}\right]$ (white diamond), $\left[\mathrm{C}_{6} \mathrm{mim}\right]\left[\mathrm{MeSO}_{4}\right]$ (white circle), $\left[\mathrm{C}_{2} \mathrm{mim}\right]\left[\mathrm{BF}_{4}\right]$ (black square), $\left[\mathrm{C}_{3}\right.$ mim $]\left[\mathrm{BF}_{4}\right]$ (black up-pointing triangle), $\left[\mathrm{C}_{2} \mathrm{mim}\right]\left[\mathrm{NTf}_{2}\right]$ (plus sign), $\left[\mathrm{C}_{6} \mathrm{mim}\right]\left[\mathrm{BF}_{4}\right]$ (black diamond), and $\left[\mathrm{C}_{4} \mathrm{mim}\right][$ Triflate $]$ (multiplication sign), and solid lines are those predicted by the proposed model

where $\eta^{*}$ is reduced viscosity and $\eta$ is dynamic viscosity in Pas units. $\eta_{S H S-M E T}{ }^{*}$ is the relevant expression to viscosity from smooth hard sphere-modified Enskog's theory, viz.:

$$
\eta_{S H S-M E T}^{*}=4 y\left[\frac{1}{\left(Z_{M V}^{H S}-1\right)}+0.8+0.761\left(Z_{M V}^{H S}-1\right)\right]
$$

In this study, $C_{\eta}$ is proposed to be expressed in terms of universal function of reduced pressure $\left(P_{r}=P / 0.1 \mathrm{MPa}\right)$

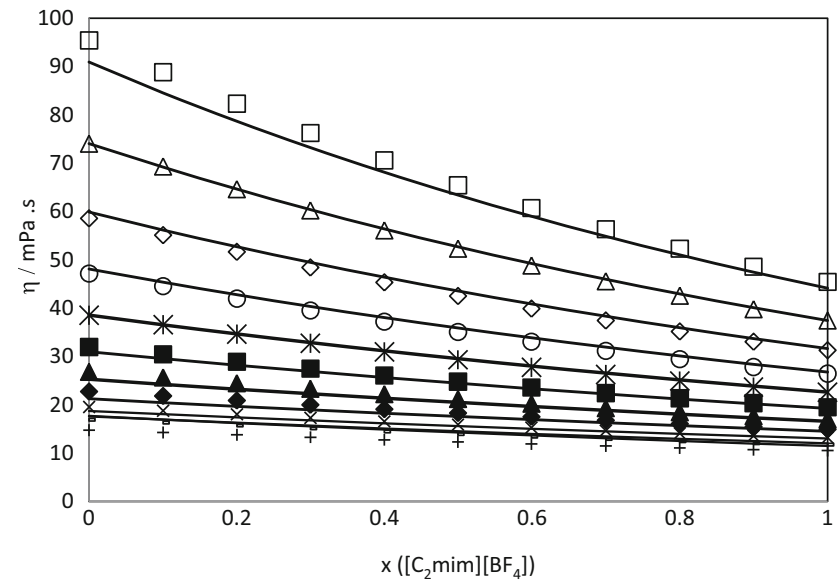

Fig. 3 Viscosity of mixtures of $x\left[\mathrm{C}_{2} \mathrm{mim}\right]\left[\mathrm{BF}_{4}\right]+(1-x)\left[\mathrm{C}_{3} \mathrm{~min}\right]\left[\mathrm{BF}_{4}\right]$ in terms of mole fraction. Symbols refer to experimental values [39] at $293.15 \mathrm{~K}$ (white square), $298.15 \mathrm{~K}$ (white up-pointing triangle), $303.15 \mathrm{~K}$ (white diamond), $308.15 \mathrm{~K}$ (white circle), $313.15 \mathrm{~K}$ (asterisk), $318.15 \mathrm{~K}$ (black square), $323.15 \mathrm{~K}$ (black up-pointing triangle), $328.15 \mathrm{~K}$ (black diamond), $333.15 \mathrm{~K}$ (multiplication sign), $338.15 \mathrm{~K}$ (white square), and $343.15 \mathrm{~K}$ (plus sign), while the solid curves represent the results calculated with the proposed modeling atmospheric pressure 


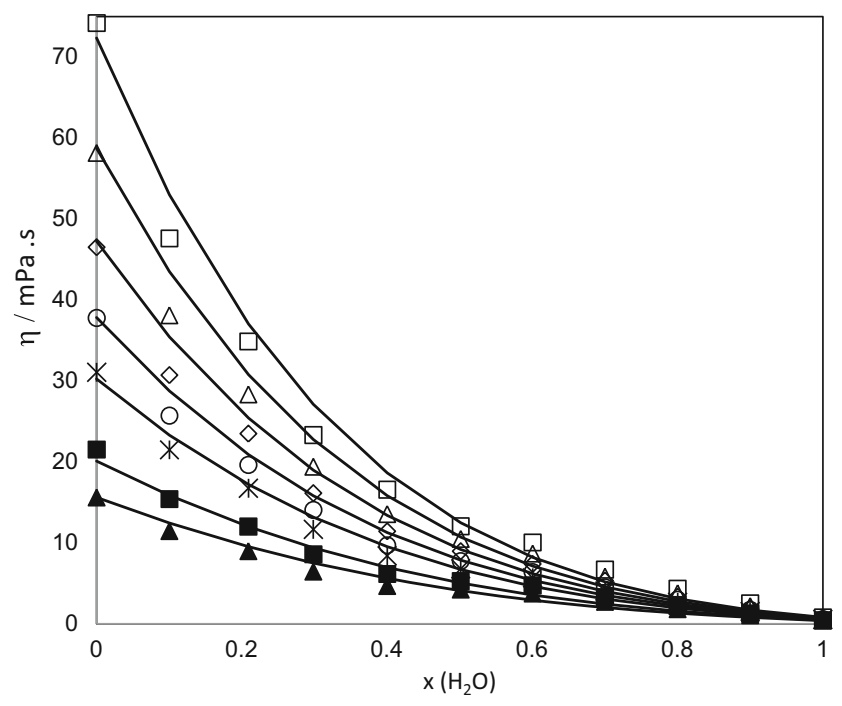

Fig. 4 Viscosity of $x\left[\mathrm{H}_{2} \mathrm{O}\right]+(1-x)\left[\mathrm{C}_{4} \min \right][\mathrm{BF} 4]$. Symbols refer to experimental values [33] at $303.15 \mathrm{~K}$ (white square), $308.15 \mathrm{~K}$ (white uppointing triangle), $313.15 \mathrm{~K}$ (white diamond), $318.15 \mathrm{~K}$ (white circle), $323.15 \mathrm{~K}$ (asterisk), $333.15 \mathrm{~K}$ (black square), and $343.15 \mathrm{~K}$ (black uppointing triangle), while the solid curves represent the results calculated with the proposed modeling atmospheric pressure

as follows:

$C_{\eta}=\exp \left(6.4 \times 10^{-4}\left(P_{r}-1\right)\right)$

In Eq. (19), $F_{\eta}$ is a temperature-dependent function which is expressed in terms of the reduced temperature $\left(T_{r}=k_{B} T / \varepsilon\right)$ as follows:

$F_{\eta}\left(k_{B} T / \varepsilon\right)=c_{1}+c_{2} T_{r}+c_{3} T_{r}^{3}$

where $k_{B} T$ is thermal energy per one molecule. The coefficients $c_{1}-c_{3}$ were fixed for each IL by correlating the viscosity data at $0.1 \mathrm{MPa}$ and their numerical values were reported in Table 4.

Table 5 contains the AAD of the calculated viscosity coefficients of studied pure ILs from the experimental ones $[33,34,37,39,41-48]$ in temperature interval $278-363 \mathrm{~K}$ and atmospheric pressure. For 150 data point examined, the overall AADs for MET and RHS models were found to be 5.78 and 6.06 , respectively. Further, Fig. 2 displays the predicted viscosity of several pure ILs in terms of temperature in atmospheric pressure using the MET method. Our results are in accord with those given in literature. It should be mentioned that the errors associated with the calculated viscosity using our model are of the order of \pm 26.40 .

\section{Extension to mixtures}

It is interesting to check further the present model by predicting the viscosity of mixtures. In this regard, the ideal Grunberg and Nissan mixing law [49] was employed to predict the mixture viscosity of ionic liquids as follows:

$$
\begin{aligned}
\log _{10}\left(\eta_{m}\right)= & x_{1} \log _{10}\left(\eta_{1}\right)+x_{2} \log _{10}\left(\eta_{2}\right) \\
& +2 x_{1} x_{2} k_{12}\left(\frac{M_{w, 1} M_{w, 2}}{M_{w, m}}\right)^{1 / 2}
\end{aligned}
$$

where $\eta_{m}$ represents the absolute viscosity of the mixture, $x_{i}$ is the mole fraction of component $\mathrm{i}$, and $\eta_{i}$ is the absolute viscosity of pure component $i . k_{12}$ is an interaction parameter.

Figures 3 and 4 represent the calculated viscosity of binary mixtures formed by ILs in terms of mole fraction and at different temperatures and atmospheric

\begin{tabular}{|c|c|c|c|c|c|c|}
\hline & $T_{\min }-T_{\max }(\mathrm{K})$ & NP & $\mathrm{AAD}^{\mathrm{a}}$ & $\mathrm{AAD}^{\mathrm{b}}$ & $k_{12}$ & Ref. \\
\hline $\left.\mathrm{C}_{2} \mathrm{mim}\right]\left[\mathrm{BF}_{4}\right]+\left[\mathrm{C}_{3} \mathrm{mim}\right]\left[\mathrm{BF}_{4}\right]$ & $293-343$ & 121 & 2.41 & 2.40 & $3 \times 10^{-5}$ & [39] \\
\hline$\left[\mathrm{C}_{2} \mathrm{mim}\right]\left[\mathrm{BF}_{4}\right]+\left[\mathrm{C}_{6} \mathrm{mim}\right]\left[\mathrm{BF}_{4}\right]$ & $293-343$ & 121 & 6.39 & 3.62 & 0.00497 & [39] \\
\hline$\left[\mathrm{C}_{3} \mathrm{mim}\right]\left[\mathrm{BF}_{4}\right]+\left[\mathrm{C}_{6} \mathrm{mim}\right]\left[\mathrm{BF}_{4}\right]$ & $293-343$ & 121 & 6.39 & 4.38 & 0.00416 & [39] \\
\hline$\left[\mathrm{C}_{4} \mathrm{mim}\right]\left[\mathrm{BF}_{4}\right]+$ water & $303-343$ & 77 & 29.32 & 13.65 & 0.0760 & [33] \\
\hline$\left[\mathrm{C}_{4} \mathrm{mim}\right]\left[\mathrm{C}(\mathrm{CN})_{3}\right]+$ water & $278-358$ & 81 & 33.04 & 15.08 & 0.112 & [37] \\
\hline$\left[\mathrm{C}_{4} \mathrm{mim}\right]\left[\mathrm{N}(\mathrm{CN})_{2}\right]$ +water & $278-358$ & 63 & 36.55 & 11.74 & 0.113 & [37] \\
\hline$\left[\mathrm{C}_{4} \mathrm{mim}\right][$ Triflate $]+$ water & $303-343$ & 77 & 43.28 & 17.64 & 0.143 & {$[34]$} \\
\hline Overall & & 661 & 22.48 & 9.79 & & \\
\hline
\end{tabular}

Table 6 AAD $(\%)$ of the calculated viscosity of binary IL-IL and IL-solvents using the present model compared with the experiment [33, 34, 37, 39]

$\mathrm{AAD}=100 / \mathrm{NP} \sum_{i=1}^{\mathrm{NP}}\left|\eta_{m, i}^{\text {Calc. }}-\eta_{m, i}^{\mathrm{Lit}}\right| / \eta_{m, i}^{\mathrm{Lit}}$

$N P$ number of literature data points examined

${ }^{\text {a }}$ Calculations have been performed with $k_{12}$ equal to zero

${ }^{\mathrm{b}}$ Calculations have been performed with non-zero $k_{12}$ 
pressure. The results show that the model is able to describe liquid viscosity data for binary mixture of ILs. We have calculated the viscosities of binary mixtures of ILs over the whole range of concentration. Further, Table 6 shows the AAD of the calculated viscosities of mixtures of several ionic liquids from the experimental ones [33, $34,37,39]$. From 661 data points examined for the aforementioned mixtures, the mean AAD of the predicted viscosities from measurement was found to be $22.48 \%$ and 9.79 without and with binary interaction parameter. The pleasure harmony between the results and those obtained from literature confirms the reliability of our model. Figure 5 represents the predicted viscosity of three ILs at $298.15 \mathrm{~K}$ (a-plot) and viscosity of $\left[\mathrm{C}_{6} \mathrm{mim}\right]\left[\mathrm{BF}_{4}\right]$ predicted at three temperatures $293.15,313.15$, and $333.15 \mathrm{~K}$ up to $20 \mathrm{MPa}$ (b-plot). The markers represent the experimental viscosity [50-52] and the solid lines are those obtained from the proposed MET model. As Fig. 5
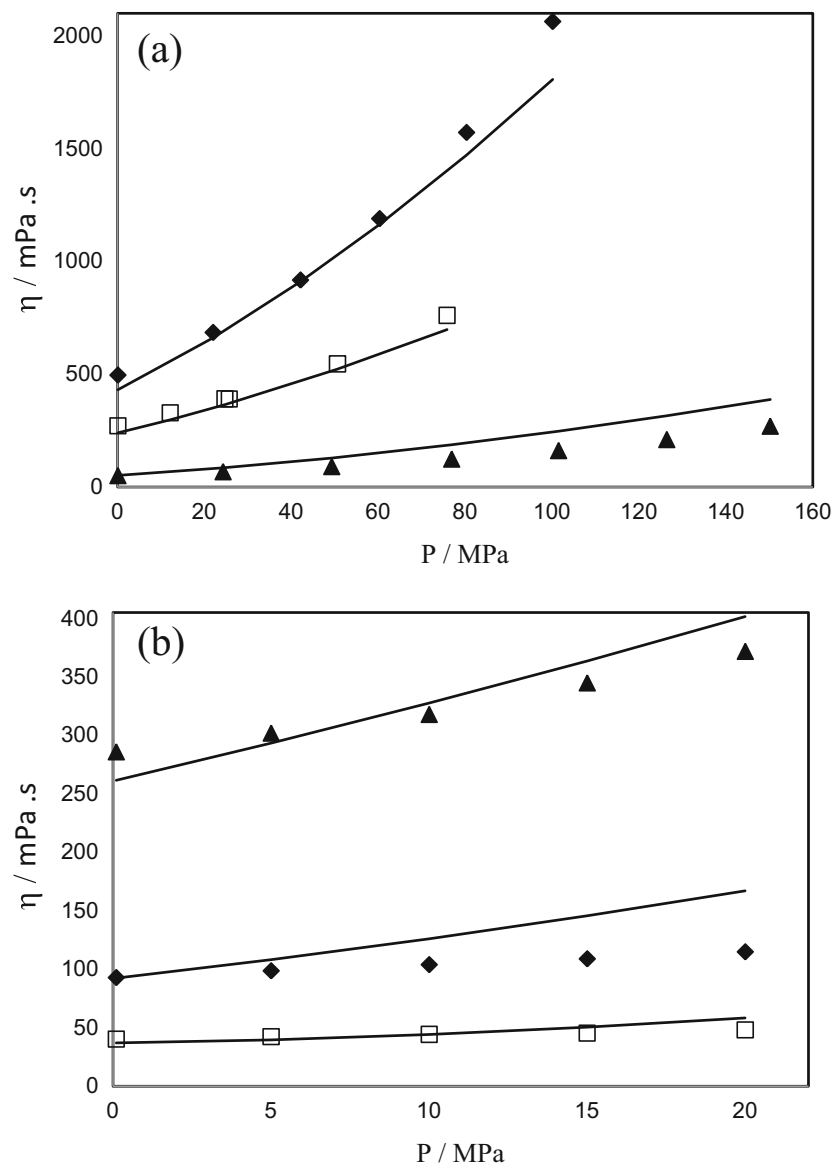

Fig. 5 The viscosity of some selected ionic liquids in terms of pressure. The markers represent the literature data $[51,52]$ for $\left[\mathrm{C}_{4} \mathrm{mim}\right]\left[\mathrm{PF}_{4}\right]$ (white square), $\left[\mathrm{C}_{4} \mathrm{mim}\right]\left[\mathrm{NTf}_{2}\right]$ (black up-pointing triangle), $\left[\mathrm{C}_{6} \mathrm{mim}\right]\left[\mathrm{PF}_{6}\right]$ and (black diamond) at $298.15 \mathrm{~K}$ (a-plot). b-plot represents the viscosity of $\left[\mathrm{C}_{6} \mathrm{mim}\right]\left[\mathrm{BF}_{4}\right]$ in terms of pressure. The markers represent the literature data [50] at temperatures $293.15 \mathrm{~K}$ (black up-pointing triangle), $313.15 \mathrm{~K}$ (black diamond), and $333.15 \mathrm{~K}$ (white square). Solid lines are those predicted by the MET model illustrates, the viscosity of IL mixture can be shown well by the present model.

Finally, we assessed the proposed MET model by studying the excess viscosity of IL-IL and IL-solvent systems which is defined as:

$\Delta \eta=\eta_{m}-\sum_{i}^{2} x_{i} \eta_{i}$

The excess behaviors of two selected IL-IL and IL-solvent systems were predicted using the proposed MET model, and the results were compared with those obtained from experimental mixture viscosity data [34, 39]. The outcomes of the computations are shown in Fig. 6.
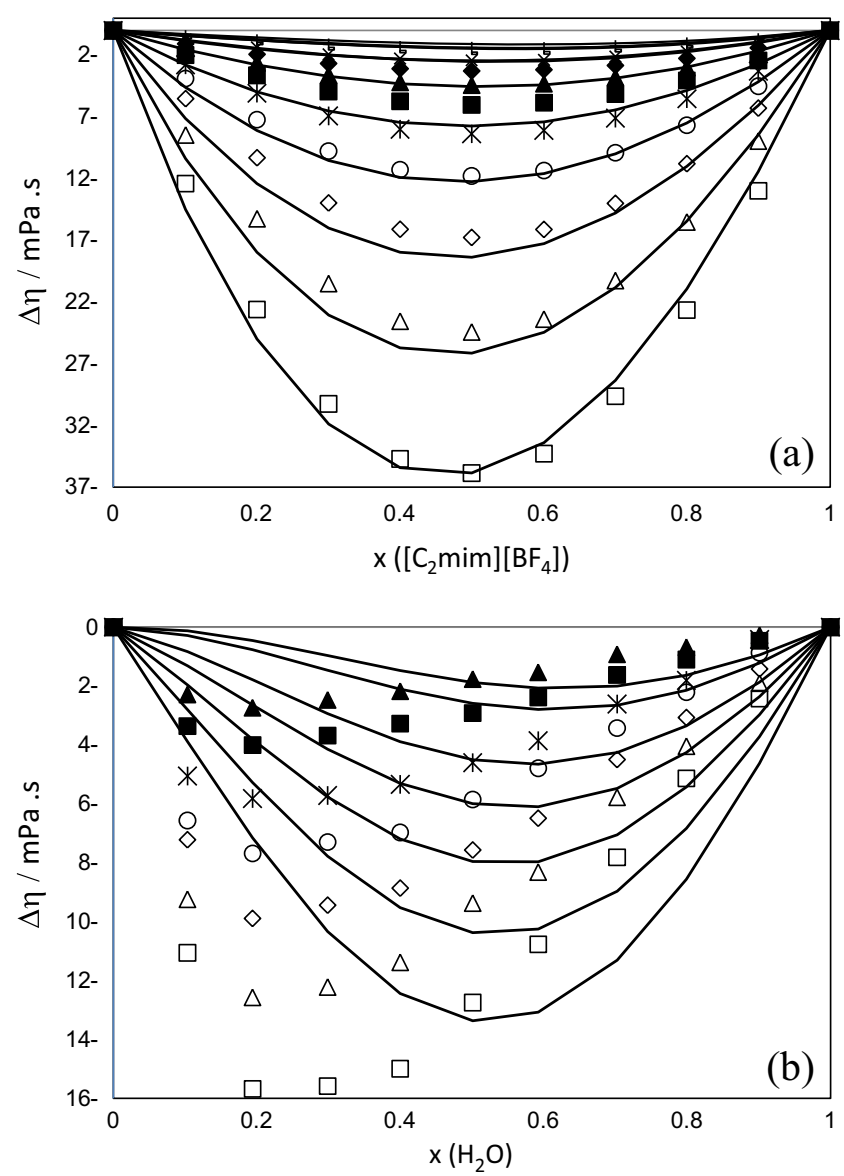

Fig. 6 Excess viscosity for the $x\left[\mathrm{C}_{2} \operatorname{mim}\right]\left[\mathrm{BF}_{4}\right]+(1-x)\left[\mathrm{C}_{6} \mathrm{~min}\right]\left[\mathrm{BF}_{4}\right]$ in terms of mole fraction. Symbols refer to experimental values [39] for temperatures $293.15 \mathrm{~K}$ (white square), $298.15 \mathrm{~K}$ (white up-pointing triangle), $303.15 \mathrm{~K}$ (white diamond), $308.15 \mathrm{~K}$ (white circle), $313.15 \mathrm{~K}$ (asterisk), $318.15 \mathrm{~K}$ (black square), $323.15 \mathrm{~K}$ (black up-pointing triangle), $328.15 \mathrm{~K}$ (black diamond), $333.15 \mathrm{~K}$ (multiplication sign), $338.15 \mathrm{~K}$ (white square), and $343.15 \mathrm{~K}$ (plus sign) (a-plot). b-plot represents the excess viscosity of $x\left[\mathrm{H}_{2} \mathrm{O}\right]+(1-x)\left[\mathrm{C}_{4} \mathrm{~min}\right][$ Triflate $]$. Symbols refer to experimental values [34] at temperatures $303.15 \mathrm{~K}$ (white square), $308.15 \mathrm{~K}$ (white up-pointing triangle), $313.15 \mathrm{~K}$ (white diamond), $318.15 \mathrm{~K}$ (white circle), $323.15 \mathrm{~K}$ (asterisk), $333.15 \mathrm{~K}$ (black square), and $343.15 \mathrm{~K}$ (black up-pointing triangle). The solid curves represent the results calculated with the MET model in atmospheric pressure 


\section{Surface tension}

Due to the fact that temperature-dependent surface tension of fluids (below their critical temperatures) are perfectly linear and according to the successful applicability of temperature dependence of viscosity [53], we propose that the surface tension can be related to the fluidity as,

$\ln \gamma=C+D\left(\frac{1}{\eta}\right)^{\phi}$

where $C$ and $D$ are substance-dependent constants and, for studied ILs, constants $C$ and $D$ are reported in Table 7 . The exponent $\phi=0.30$ is used in Eq. (25) to fit the imidazoliumbased ILs with simple anion content using the experimental viscosity [41] and surface tension [54] data.

In Fig. 7, the surface tension in terms of temperature has been provided and compared with literature data [42, 45, 46, $54,55]$. The results show that the model is able to describe surface tension data for pure ILs.

To assess further the degree of accuracy of the proposed model, we compare the AADs of our calculations with those obtained from other methods. In Table 7, we have listed the AAD of the predicted surface tension for 81 data points using the present model, the results obtained from the Alavianmehr et al. [56], quantitative structure-property relationship (QSPR) strategy [57], and corresponding states group contribution (CS-GC) method [58] from the literature data [42, 45,

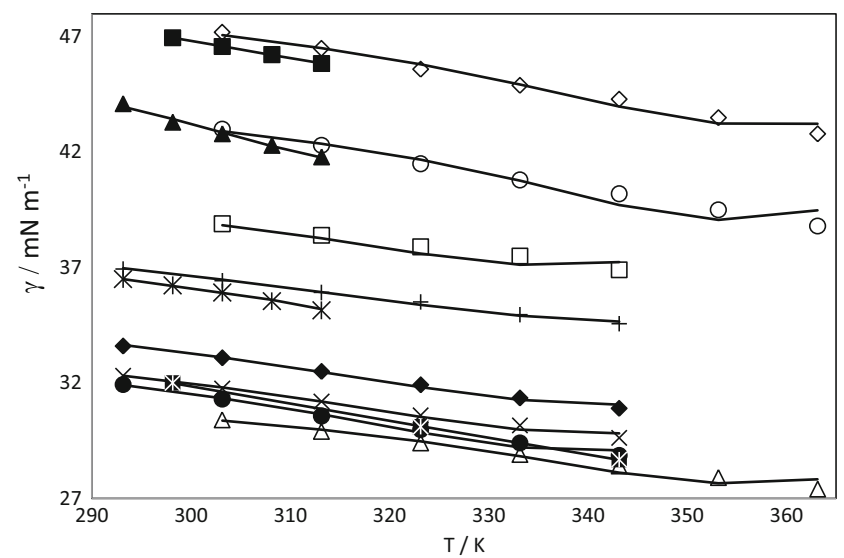

Fig. 7 The calculated surface tension of some selected ionic liquids as a function of temperature. The markers represent the literature data $[42,45$, 54, 55], $\left[\mathrm{C}_{6} \mathrm{mim}\right]\left[\mathrm{BF}_{4}\right]$ (white square), $\left[\mathrm{C}_{8} \mathrm{mim}\right]\left[\mathrm{BF}_{4}\right]$ (white up-pointing triangle), $\left[\mathrm{C}_{4} \mathrm{mim}\right]\left[\mathrm{PF}_{6}\right]$ (white diamond), $\left[\mathrm{C}_{6} \mathrm{mim}\right]\left[\mathrm{PF}_{6}\right]$ (white circle), $\left[\mathrm{C}_{8} \mathrm{mim}\right]\left[\mathrm{PF}_{6}\right]$ (asterisk), $\left[\mathrm{C}_{2} \mathrm{mim}\right]\left[\mathrm{EtSO}_{4}\right]$ (black square), $\left[\mathrm{C}_{4} \mathrm{mim}\right]\left[\mathrm{MeSO}_{4}\right]$ (black up-pointing triangle), $\left[\mathrm{C}_{2} \mathrm{mim}\right]\left[\mathrm{NTf}_{2}\right]$ (plus sign), $\left[\mathrm{C}_{4} \mathrm{mim}\right]\left[\mathrm{NTf}_{2}\right]$ (black diamond), $\left[\mathrm{C}_{6} \mathrm{mim}\right]\left[\mathrm{NTf}_{2}\right]$ (multiplication sign), $\left[\mathrm{C}_{8} \mathrm{mim}\right]\left[\mathrm{NTf}_{2}\right]$ (black circle), and $\left[\mathrm{C}_{10} \mathrm{mim}\right]\left[\mathrm{NTf}_{2}\right]$ (wingdings 181 ), and solid lines are those predicted by the proposed model in atmospheric pressure

$46,54,55]$. The interesting point of the present study is that the AADs of the calculated surface tension from the present model are remarkably lower than those obtained from other methods. The overall AAD of the predicted surface tension for 81 data points using the present model, Alavianmehr et al., QSPR, and CS-GC methods were found to be $0.29,1.45$,

Table 7 The scaling constants to be used in Eq. (25)and AAD (\%) of the calculated surface tension using the present model and those obtained by Alavianmehr et al. [56], QSPR [57] strategy, and CS-GC [58] method

\begin{tabular}{|c|c|c|c|c|c|c|c|c|c|}
\hline & $C$ & $-D$ & $T_{\min }-T_{\max }(\mathrm{K})$ & NP & This work & Alavianmehr et al. & QSPR & $\mathrm{CS}-\mathrm{GC}$ & Ref. \\
\hline$\left[\mathrm{C}_{4} \mathrm{mim}\right]\left[\mathrm{BF}_{4}\right]$ & 3.86 & 0.348 & $303-343$ & 07 & 0.12 & 2.09 & 9.48 & - & [54] \\
\hline$\left[\mathrm{C}_{6} \mathrm{mim}\right]\left[\mathrm{BF}_{4}\right]$ & 3.74 & 0.375 & $303-363$ & 05 & 0.64 & - & - & - & [54] \\
\hline$\left[\mathrm{C}_{8} \mathrm{mim}\right]\left[\mathrm{BF}_{4}\right]$ & 3.51 & 0.491 & $303-363$ & 07 & 0.61 & 3.81 & 15.69 & 6.14 & [54] \\
\hline$\left[\mathrm{C}_{4} \mathrm{mim}\right]\left[\mathrm{PF}_{6}\right]$ & 3.95 & 0.464 & $303-363$ & 07 & 0.45 & 2.03 & 2.45 & - & {$[54]$} \\
\hline$\left[\mathrm{C}_{6} \mathrm{mim}\right]\left[\mathrm{PF}_{6}\right]$ & 3.85 & 0.505 & $303-363$ & 07 & 0.71 & 0.23 & 6.38 & - & {$[54]$} \\
\hline$\left[\mathrm{C}_{8} \mathrm{mim}\right]\left[\mathrm{PF}_{6}\right]$ & 3.85 & 0.505 & $293-313$ & 05 & 0.087 & 1.79 & 11.93 & - & [45] \\
\hline$\left[\mathrm{C}_{2} \mathrm{mim}\right]\left[\mathrm{EtSO}_{4}\right]$ & 3.96 & 0.428 & $298-313$ & 04 & 0.021 & - & - & - & [45] \\
\hline$\left[\mathrm{C}_{4} \mathrm{mim}\right]\left[\mathrm{MeSO}_{4}\right]$ & 3.92 & 0.735 & $293-313$ & 05 & 0.18 & - & - & - & [42] \\
\hline$\left[\mathrm{C}_{10} \mathrm{mim}\right]\left[\mathrm{NTf}_{2}\right]$ & 3.62 & 0.631 & $298-343$ & 03 & 0.082 & - & - & - & {$[55]$} \\
\hline$\left[\mathrm{C}_{2} \mathrm{mim}\right][$ Triflat $]$ & 3.87 & 0.229 & $278-338$ & 07 & 0.062 & - & - & - & {$[55]$} \\
\hline$\left[\mathrm{C}_{8} \mathrm{mim}\right]\left[\mathrm{NTf}_{2}\right]$ & 3.59 & 0.532 & $293-343$ & 06 & 0.38 & - & - & - & {$[55]$} \\
\hline$\left[\mathrm{C}_{6} \mathrm{mim}\right]\left[\mathrm{NTf}_{2}\right]$ & 3.59 & 0.447 & $293-343$ & 06 & 0.27 & 0.21 & 6.59 & 5.20 & [55] \\
\hline$\left[\mathrm{C}_{4} \mathrm{mim}\right]\left[\mathrm{NTf}_{2}\right]$ & 3.64 & 0.447 & $293-343$ & 06 & 0.23 & 1.00 & 3.98 & 3.23 & [55] \\
\hline$\left[\mathrm{C}_{2} \mathrm{mim}\right]\left[\mathrm{NTf}_{2}\right]$ & 3.73 & 0.376 & $293-343$ & 06 & 0.17 & 0.42 & 5.33 & 2.00 & {$[55]$} \\
\hline Overall & & & & 81 & 0.29 & 1.45 & 7.73 & 4.14 & \\
\hline
\end{tabular}

$\mathrm{AAD}=100 / \mathrm{NP} \sum_{i=1}^{\mathrm{NP}}\left|\gamma_{i}^{\text {Calc. }}-\gamma_{i}^{\text {Lit. }}\right| / \gamma_{i}^{\text {Lit. }}$

$N P$ number of literature data points examined 
7.73, and 4.14\%, respectively. As we can see from Table 7, the present model is the most accurate among other abovementioned methods. It should be mentioned that the error propagation uncertainty of calculated surface tension was of the order of \pm 1.73 .

\section{Conclusion}

We successfully applied the PHTS EOS to the mixture of ILs using alternative corresponding states correlations for two temperature-dependent parameters that appeared in the EOS. When tested against experimental data, the calculated liquid densities for binary mixtures including $\mathrm{IL}+$ solvent and $\mathrm{IL}+$ IL gave the mean average absolute deviations of $0.44 \%$ for 1927 data points. Also, we showed that the liquid densities and molecular parameters taken from previously developed EOS were adequately rational for predicting the densities of mixture involving ILs using some simple mixing rules.

The thermophysical properties such as viscosity and surface tension were predicted by the present model. When compared with other works and also with literature data, the accuracy of our model was further revealed. The predicted viscosity coefficient and surface tension of ionic liquids were in good agreement with experimental data.

Finally, the non-additivity behavior of the studied mixtures was also investigated. The sign of the non-additivity parameter indicates a tendency toward attraction between the dissimilar molecules in the mixture. However, the value of this parameter was not large, which implied that the hard-sphere model is able to model the excess properties of the present mixtures. However, considering the non-additivity effect in our calculations showed that this effect can improve slightly the results of mixture densities.

Acknowledgements We are grateful to the research committee of the Shiraz University of Technology for supporting this project.

\section{Nomenclature}

AAD; Average absolute deviation (\%),g; Pair radial distribution function of hard-spheres at contact $(\mathrm{m}), b(T)$; van der Waals covolume, $\mathrm{m}^{3}, P$; Pressure, $\mathrm{Pa}, R$; Gas constant, $\mathrm{J} / \mathrm{mol} \mathrm{K}, T$; Absolute temperature, $\mathrm{K} ; k$, ${ }_{B}$; Boltzmann's constant, $\mathrm{J} / \mathrm{K} M_{\mathrm{w}}$; Molecular weight, $c_{1}-c_{3}$; Coefficients, $N_{a}$; Avogadro's number, mol, $F_{\eta}$; Temperature-dependent function, $C_{\eta}$; Coupling parameters, $a(T)$; Strengths of attractive forces, $\mathrm{J} \mathrm{m}^{-3}$

\section{$F_{a}$ and $F_{b}$ Universal functions}

$y$; Packing fraction, $V$; Molar volume, $C$; Substance-dependent constant, $D$; Substance-dependent constant, $M$; Thermodynamic properties, $Z$; Compressibility factor, $x$; Molar fraction

\section{Greek letters}

$\varepsilon$; Non-bonded interaction energy parameter, $\mathrm{J}, \sigma$; Effective hard-sphere diameter, nm, $\eta$; Viscosity, mPa s, $\eta^{*}$; Reduced viscosity, $\rho$; Molar density, $\mathrm{mol} / \mathrm{m}^{3}, \alpha$; Adjustable parameter, $\beta$; Adjustable parameter, $\omega$; Adjustable parameter, $\phi$; Exponent, $\gamma$; Surface tension, $\mathrm{mN} \mathrm{m}^{-1}, \pi$; pi $=3.14, \lambda$; Adjustable parameter

\section{Superscript}

Li; Literature, Calc; Calculated, $E$; Excess, $H^{3}$; Hard-trimer-chain reference system, Pert; Perturbed, HS; Hard-sphere, RHS; Rough hard-sphere

\section{Subscript}

MV; Malijevsky-Veverka, $m$; Mixture

Open Access This article is distributed under the terms of the Creative Commons At $\mathrm{t} \mathrm{r}$ ibut ion 4.0 Inte rnat ional $\mathrm{Li}$ cense (ht tp: // creativecommons.org/licenses/by/4.0/), which permits unrestricted use, distribution, and reproduction in any medium, provided you give appropriate credit to the original author(s) and the source, provide a link to the Creative Commons license, and indicate if changes were made.

\section{References}

1. Ficke LE, Novak RR, Brennecke JF (2010) Thermodynamic and thermophysical properties of ionic liquid + water systems. J Chem Eng Data 55:4946-4950

2. Palomar J, Ferro VR, Torrecilla JS, Rodríguez F (2007) Density and molar volume predictions using COSMO-RS for ionic liquids. An approach to solvent design. Ind Eng Chem Res 46:6041-6048

3. Gardas RL, Coutinho JA (2008) Extension of the Ye and Shreeve group contribution method for density estimation of ionic liquids in a wide range of temperatures and pressures. Fluid Phase Equilib 263:26-32

4. Valderrama JO, Reategui A, Rojas RE (2009) Density of ionic liquids using group contribution and artificial neural networks. Ind Eng Chem Res 48:3254-3259

5. Aparicio S, Atilhan M, Karadas F (2010) Thermophysical properties of pure ionic liquids: review of present situation. Ind Eng Chem Res 49:9580-9595

6. Hosseini SM (2010) A perturbed hard-sphere equation of state for phosphonium-, pyridinium-, and pyrrolidinium-based ionic liquids. Ionics 16:571-575

7. Hosseini SM, Moghadasi J, Papari MM (2010) A perturbed hardsphere equation of state extended to imidazolium-based ionic liquids. Ionics 16:757-761

8. Hosseini SM, Moghadasi J, Papari MM, Nobandegani FF (2011) Modeling the volumetric properties of mixtures involving ionic liquids using perturbed hard-sphere equation of state. J Mol Liq 160:67-71

9. Hosseini SM, Moghadasi J, Papari MM, Fadaei Nobandegani F (2012) Modeling the volumetric properties of ionic liquids using modified perturbed hard-sphere equation of state: application to pure and binary mixtures. Ind Eng Chem Res 51:758-766

10. Papari MM, Amighi S, Kiani M, Mohammad-Aghaie D, Haghighi B (2012) Modification of a statistical mechanically-based equation of state: application to ionic liquids. J Mol Liq 175:61-66

11. Papari MM, Hosseini SM, Fadaei-Nobandegani F, Moghadasi J (2012) Modeling of P- $\rho$-T properties of ionic liquids using ISM equation of state: application to pure component and binary mixtures. Korean J Chem Eng 29:1628-1637

12. Hosseini SM, Alavianmehr MM, Moghadasi J (2013) Density and isothermal compressibility of ionic liquids from perturbed harddimer-chain equation of state. Fluid Phase Equilib 356:185-192

13. Alavianmehr MM, Hosseini SM, Amighi S (2014) Volumetric properties of ionic liquids and their binary mixtures from improved Tao-Mason equation of state. J Mol Liq 191:166-171 
14. Alavianmehr MM, Taghizadehfard M, Hosseini SM (2016) Development of a perturbed hard-sphere equation of state for pure and mixture of ionic liquids. Ionics 22:649-660

15. Alavianmehr MM, Hosseini SM, Akbari F (2015) Modeling volumetric properties of amorphous and molten polymers using new perturbed-chain equation of state. J Mol Liq 212:900-906

16. Alavianmehr MM, Akbari F, Behjatmanesh-Ardakani R (2016) Modelling phase equilibria of pure ionic liquids from a new equation of state. Ionics 22(12):2447-2459

17. Hsu CC, Eyring H (1972) Significant liquid-structure theory of viscosity and self-diffusion of the alkali metals. Proc Nat Acad Sci USA 69:1342-1345

18. Skomorokhov VI, Dregalin AF (1993) A method for calculating the temperature dependence of the viscosity of low-molecular-weight organic liquids. J Eng Phys Thermophys 65:1044-1046

19. Swain N, Singh SK, Panda D, Chakravortty V (2000) Viscosities and densities of ternary liquid mixtures of DEHPA, benzene and ortho-xylene. J Mol Liq 85:321-330

20. Meng X, Zheng P, Wu J, Liu Z (2008) Density and viscosity measurements of diethyl ether from 243 to $373 \mathrm{~K}$ and up to $20 \mathrm{MPa}$. Fluid Phase Equilib 271:1-5

21. Hirschfelder J, Bird RB, Curtiss CF (1964) Molecular theory of gases and liquids, 2nd edn. Wiley, New York

22. Hosseini SM, Alavianmehr MM, Moghadasi J (2016) Transport properties of pure and mixture of ionic liquids from new rough hard-sphere-based model. Fluid Phase Equilib 429:266-274

23. Grigoryev BA, Nemzer BV, Kurumov DS, Sengers JV (1992) Surface tension of normal pentane, hexane, heptane, and octane. Int J Thermophys 13:453-464

24. Miqueu C, Broseta D, Satherley J, Mendiboure B, Lachaise J, Graciaa A (2000) An extended scaled equation for the temperature dependence of the surface tension of pure compounds inferred from an analysis of experimental data. Fluid Phase Equilib 172:169-182

25. Ghatee MH, Soorghali A (2006) Application of extended scaling law to the surface tension of fluids of wide range of molecular shapes. Fluid Phase Equilib 249:153-158

26. Restolho J, Serro AP MJL, Saramago B (2009) Viscosity and surface tension of 1-ethanol-3-methylimidazolium tetrafluoroborate and 1-methyl-3-octylimidazolium tetrafluoroborate over a wide temperature range. J Chem Eng Data 54:950-955

27. Moldover MR (1985) Interfacial tension of fluids near critical points and two-scale-factor universality. PhysRev A31:10221033

28. Ghatee MH, Maleki A, Ghaed-Sharaf H (2003) Extended generic nature of surface entropy. Langmuir 19:211-213

29. Srivastava V, Khanna KN (2002) Equation of state of long chain molecules and rings. Mol Phys 100:311-316

30. Malijevský A, Veverka J (1999) New equations of state for pure and binary hard-sphere fluids. Phys Chem Chem Phys 1:4267-4270

31. Boublík T (1970) Hard-sphere equation of state. J Chem Phys 53: 471-472

32. Navia P, TroncosoJ RL (2007) Excess magnitudes for ionic liquid binary mixtures with a common ion. J Chem Eng Data 52:1369-1374

33. Zhou Q, Wang LS, Chen HP (2006) Densities and viscosities of 1-butyl-3-methylimidazolium tetrafluoroborate $+\mathrm{H}_{2} \mathrm{O}$ binary mixtures from (303.15 to 353.15$) \mathrm{K}$. J Chem Eng Data 51: 905-908

34. Ge ML, Zhao RS, Yi YF, Zhang Q, Wang LS (2008) Densities and viscosities of 1-butyl-3-methylimidazolium trifluoromethanesulfonate $+\mathrm{H}_{2} \mathrm{O}$ binary mixtures at $\mathrm{T}=(303.15$ to 343.15$) \mathrm{K}$. J Chem Eng Data 53:2408-2411

35. Lehmann J, Rausch MH, Leipertz A, Fröba AP (2010) Densities and excess molar volumes for binary mixtures of ionic liquid 1- ethyl-3-methylimidazolium ethylsulfate with solvents. J Chem Eng Data 55:4068-4074

36. Vercher E, Orchillés AV, Miguel PJ, Martínez-Andreu A (2007) Volumetric and ultrasonic studies of 1-ethyl-3-methylimidazolium trifluoromethanesulfonate ionic liquid with methanol, ethanol, 1propanol, and water at several temperatures. J Chem Eng Data 52: $1468-1482$

37. Carvalho PJ, Regueira T, Santos LM, FernandezJ CJA (2010) Effect of water on the viscosities and densities of 1-butyl-3-methylimidazolium dicyanamide and 1-butyl-3-methylimidazolium tricyanomethane at atmospheric pressure. J Chem Eng Data 55:645-652

38. Zafarani-Moattar MT, Majdan-Cegincara R (2007) Viscosity, density, speed of sound, and refractive index of binary mixtures of organic solvent+ ionic liquid, 1-butyl-3-methylimidazolium hexafluorophosphate at 298.15 K. J Chem Eng Data 52:2359-2364

39. Song D, Chen J (2014) Density and viscosity data for mixtures of ionic liquids with a common anion. J Chem Eng Data 59: 257-262

40. Fadaei-Nobandegani F, Hosseini SM, Papari MM, Moghadasi J (2012) Volumetric properties of mixtures involving ionic liquids from improved equation of state. Thermochim Acta 546:94-101

41. Seddon KR, Stark A, Torres MJ (2002) Viscosity and density of 1alkyl-3-methylimidazolium ionic liquids. Torres, ACS Symp Ser 819:35-49

42. Pereiro AB, Verdía P, Tojo E, Rodríguez A (2007) Physical properties of 1-butyl-3-methylimidazolium methyl sulfate as a function of temperature. J Chem Eng Data 52:377-380

43. Ahosseini A, Scurto AM (2008) Viscosity of imidazolium-based ionic liquids at elevated pressures: cation and anion effects. Int J Thermophys 29:1222-1243

44. Tokuda H, Hayamizu K, Ishii K, Susan MABH, Watanabe M (2005) Physicochemical properties and structures of room temperature ionic liquids. 2. Variation of alkyl chain length in imidazolium cation. J Phys Chem B 109:6103-6110

45. Pereiro AB, Legido JL, Rodrı A (2007) Physical properties of ionic liquids based on 1-alkyl-3-methylimidazolium cation and hexafluorophosphate as anion and temperature dependence. J Chem Thermodyn 39:1168-1175

46. Gómez E, González B, Calvar N, Tojo E, Domínguez Á (2006) Physical properties of pure 1-ethyl-3methylimidazolium ethylsulfate and its binary mixtures with ethanol and water at several temperatures. J Chem Eng Data 51:2096-2102

47. Mokhtarani B, Mojtahedi MM, Mortaheb HR, Mafi M, Yazdani F, Sadeghian F (2008) Densities, refractive indices, and viscosities of the ionic liquids 1-methyl-3octylimidazolium tetrafluoroborate and 1-methyl-3butylimidazolium perchlorate and their binary mixtures with ethanol at several temperatures. J Chem Eng Data 53:677-682

48. Rodríguez H, Brennecke JF (2006) Temperature and composition dependence of the density and viscosity of binary mixtures of water + ionic liquid. J Chem Eng Data 51:2145-2155

49. Grunberg L, Nissan AH (1949) Mixture law for viscosity. Nature 164:799-800

50. Tomida D, Kenmochi S, Tsukada T, Qiao K, Bao Q, Yokoyama C (2012) Viscosity and thermal conductivity of 1-hexyl-3methylimidazolium tetrafluoroborate and 1-octyl-3methylimidazolium tetrafluoroborate at pressures up to $20 \mathrm{MPa}$. Int J Thermophys 33(6):959-969

51. Harris KR, Woolf LA, Kanakubo M (2005) Temperature and pressure dependence of the viscosity of the ionic liquid 1-butyl-3methylimidazolium hexafluorophosphate. J Chem Eng Data 50(5):1777-1782

52. Harris KR, Kanakubo M, Woolf LA (2007) Temperature and pressure dependence of the viscosity of the ionic liquids 1-hexyl-3methylimidazolium hexafluorophosphate and 1-butyl-3- 
methylimidazolium bis(trifluoromethylsulfonyl) imide. J Chem Eng Data 52(3):1080-1085

53. Ghatee MH, Zare M, Zolghadr AR, Moosavi F (2010) Temperature dependence of viscosity and relation with the surface tension of ionic liquids. Fluid Phase Equilib 291:188-194

54. Ghatee MH, Zolghadr AR (2008) Surface tension measurements of imidazolium-based ionic liquids at liquid-vapor equilibrium. Fluid Phase Equilib 263:168-175

55. Carvalho PJ, Freire MG, Marrucho IM, Queimada AJ, Coutinho JA (2008) Surface tensions for the 1-alkyl-3-methylimidazolium bis (trifluoromethylsulfonyl) imide ionic liquids. J Chem Eng Data 53:1346-1350
56. Alavianmehr MM, Hosseini SM, Haghighi B, Moghadasi J (2015) Surface thermodynamic properties of ionic liquids from new molecular thermodynamic model and ion-contribution equation of state. Chem Eng Sci 122:622-629

57. Gardas RL, Coutinho JAP (2008) Estimation of speed of sound of ionic liquids using surface tensions and densities: a volume based approach. Fluid Phase Equilib 267:188-192

58. KJ W, Zhao CX, He CH (2012) A simple corresponding states group-contribution method for estimating surface tension of ionic liquids. Fluid Phase Equilib 328:42-48 